\title{
ON PARAMETRIZATION, OPTIMIZATION AND TRIVIALITY OF TEST CONFIGURATIONS
}

\author{
YUJI ODAKA \\ (Communicated by Lev Borisov)
}

\begin{abstract}
We give a parametrization of test configurations in the sense of Donaldson via spherical buildings, and show the existence of "optimal" destabilizing test configurations for unstable varieties, in the wake of Mumford and Kempf. We also give an account of the recent slight amendment to the definition of K-stability after $\mathrm{Li}-\mathrm{Xu}$, from two other viewpoints: from the oneparameter subgroups and from the author's blow-up formalism.
\end{abstract}

\section{INTRODUCTION}

The concept of test configurations of polarized varieties was introduced by Donaldson [Don02 to generalize K-stability after Tian [Tia97] which is a certain version of GIT stability Mum65 for polarized varieties. Indeed, to each test configuration, certain GIT weights correspond, which is a motivation of its introduction. Precisely speaking, they can be regarded as certain equivalent classes of one parameter subgroups of the general linear group $\operatorname{GL}\left(H^{0}\left(X, L^{\otimes r}\right)\right)$ for a given polarized variety $(X, L)$.

The aim of this short note is twofold. First is to point out that, roughly speaking, dividing sets of test configurations further, all equivalent classes can be parametrized by a certain simplicial complex, the so-called spherical building invented by J. Tits (cf. [Tit74]). Moreover, thanks to a theorem of Rousseau Rou78. and Kempf [Kem78], originally known as the Tits center conjecture, we conclude the existence of "optimal" destabilizing test configurations. The outcome is a geometric analogue of the Harder-Narasimhan filtrations of unstable sheaves on a fixed variety. (The analogy becomes clearer after the recent work of [GSZ11. Namely, it claims that the filtration coincides the optimal destabilizing objects in the sense of [Rou78, Kem78.) We remark here that the spherical building has already been discussed in the Mumford classic Mum65 to give applications to the stability problem, although he used the word "flag complex" with completely the same meaning. Thus, we should note that our statements are essentially consequences of [Mum65], Rou78, Kem78, with only some small arguments, so that they are not essentially new. However, it seems to the author that the results have not been recognized by the mathematical community in this area, which is one motivation for writing this note.

Received by the editors March 17, 2012 and, in revised form, February 26, 2013.

2010 Mathematics Subject Classification. Primary 14L24; Secondary 32Q20.

The author was partially supported by the Grant-in-Aid for Scientific Research (KAKENHI No. 21-3748) and the Grant-in-Aid for JSPS fellows (PD). 
The second objective of this note is to explain, from our point of view, the pathological (almost trivial but not trivial) test configurations whose existence is pointed out in LX11, subsection 2.2]. They addressed that we need to slightly modify the definition of K-stability by regarding them as trivial or "throwing them away". For details, see subsection 3.3. In that subsection, we also explain how the blow-up formalism Od11 works for that amended notion of K-stability. Moreover, the sub-locus of the spherical building which parametrizes those test configurations of a specific kind will be described as well.

\section{Statements}

We work over an arbitrary algebraically closed field $k$. We use the terminologies of line bundles and invertible sheaves interchangeably. By a polarized variety $(X, L)$, we mean that $X$ is a projective equidimensional variety and $L$ is an ample line bundle on it. At the beginning, we recall the definition of (semi-) test configurations and introduce certain (equivalence) relations for test configurations as follows.

Definition 2.1. A test configuration (resp. semi-test configuration) for a polarized variety $(X, L)$ is a quasi-projective variety $\mathcal{X}$ with an invertible sheaf $\mathcal{L}$ on it with:

(i) a $\mathbb{G}_{m}$ action on $(\mathcal{X}, \mathcal{L})$,

(ii) a projective flat morphism $p: \mathcal{X} \rightarrow \mathbb{A}^{1}$,

such that $p$ is $\mathbb{G}_{m}$-equivariant for the usual action on $\mathbb{A}^{1}$ :

$$
\begin{array}{rlr}
\mathbb{G}_{m} \times \mathbb{A}^{1} & \longrightarrow & \mathbb{A}^{1} \\
(t, x) & \longmapsto & t x,
\end{array}
$$

$\mathcal{L}$ is relatively ample (resp. relatively semi-ample), and $\left.(\mathcal{X}, \mathcal{L})\right|_{p^{-1}\left(\mathbb{A}^{1} \backslash\{0\}\right)}$ is $\mathbb{G}_{m^{-}}$ equivariantly isomorphic to $\left(X, L^{\otimes r}\right) \times\left(\mathbb{A}^{1} \backslash\{0\}\right)$ for some positive integer $r$, called an exponent, with the natural action of $\mathbb{G}_{m}$ on the latter and the trivial action on the former.

Definition 2.2. For a given polarized variety $(X, L)$, we introduce the following three pre-equivalent conditions. Two test configurations $\left(\mathcal{X}_{i}, \mathcal{L}_{i}\right)(i=1,2)$ are:

- P-pre-equivalent if $\left(\mathcal{X}_{2}, \mathcal{L}_{2}\right)$ is a base change of $\left(\mathcal{X}_{1}, \mathcal{L}_{1}\right)$ by the power morphism $t \mapsto t^{m}$ of $\mathbb{A}^{1} \rightarrow \mathbb{A}^{1}$ with some $\mathbb{Z}_{>0}$.

- Q-pre-equivalent if $\left(\mathcal{X}_{2}, \mathcal{L}_{2}\right)=\left(\mathcal{X}_{1}, \mathcal{L}_{1}^{\otimes l}\right)$ equivariantly with $l \in \mathbb{Z}_{>0}$.

- $R$-equivalent when there is a $\mathbb{G}_{m}$-equivariant isomorphism $\phi: \mathcal{X}_{1} \cong \mathcal{X}_{2}$ over $\mathbb{A}^{1}$ with $\mathcal{L}_{1} \cong \phi^{*} \mathcal{L}_{2}$. To say in other (but a little rough) words, it is exactly when they are isomorphic "except for the linearizations".

Consider an equivalent relation among test configurations which is generated by $\mathrm{P}$-pre-equivariance relation and R-equivariance relation (resp. P-pre-equivariance relation, Q-pre-equivariance relation and $\mathrm{R}$-equivalence relation) and call it $\mathrm{T}$ equivalence relation (resp. U-equivalence relation).

A T-equivalent class of test configuration $(\mathcal{X}, \mathcal{L})$ will be called a test degeneration and we write it as $\overline{(\mathcal{X}, \mathcal{L})}$. Note that, by the base change, central fiber does not change scheme-theoretically (including polarization), which is the origin of this name. Note that exponents are well-defined for each test degeneration.

A U-equivalent class of test configuration will be called a test class. Of course, a test degeneration gives a test class. 
Let us assume the polarization $L$ attached to $X$ is very ample. Then we have the following. We explain the rigorous meaning in subsections 3.1 and 3.2

Theorem 2.3. Test degenerations $\overline{(\mathcal{X}, \mathcal{L})}$ with very ample $\mathcal{L}$ of exponent 1 are parametrized by the set of rational points $\left|\Delta\left(\mathrm{GL}\left(H^{0}(X, L)\right)\right)\right|_{\mathbb{Q}}$ of the spherical building $\Delta\left(\mathrm{GL}\left(H^{0}(X, L)\right)\right)$.

Here the spherical building $\Delta\left(\mathrm{GL}\left(H^{0}(X, L)\right)\right)$ is a certain simplicial complex. Its geometric realization $\left|\Delta\left(\mathrm{GL}\left(H^{0}(X, L)\right)\right)\right|$, which we donote as $\Delta_{r}$, with a natural topology is known to have a certain natural compatible metric structure (cf. AB08, Chapter 12]). We will give the definition of the building in subsection 3.1. We will also see that although it is non-compact with respect to the usual topology, it will be compact with certain "much stuck" topology to be introduced later. Without fixing exponents, we thus also have:

Corollary 2.4. Test classes of $(X, L)$ are parametrized by

$$
\Delta_{\infty}:=\lim _{\longrightarrow}\left|\Delta\left(\mathrm{GL}\left(H^{0}\left(X, L^{\otimes r}\right)\right)\right)\right|_{\mathbb{Q}} .
$$

Here the inductive system is given by taking the power of polarization of test configurations, i.e., $(\mathcal{X}, \mathcal{L}) \rightsquigarrow\left(\mathcal{X}, \mathcal{L}^{\otimes l}\right)$ with $l \in \mathbb{Z}_{>0}$. Note that these $\Delta_{r}$ and $\Delta_{\infty}$ can be expected to correspond to the space of geodesics of (finite-dimensional or infinite-dimensional) symmetric spaces.

For the basics of stability, we refer to [Mum65], Od11]. Based on the continuous (and piecewise-linear) behavior of GIT weights [Mum65, Prop 2.14], we see the continuity of normalized Chow weights:

Proposition 2.5. The normalized Chow weights form continuous functions on $\left|\Delta\left(\mathrm{GL}\left(H^{0}\left(X, L^{\otimes r}\right)\right)\right)\right|_{\mathbb{Q}}$ with respect to the usual topology. On the other hand, it is a lower semi-continuous function with regard to the "stuck" topology.

For the definitions of stuck topology and normalized Chow weights, see subsection 3.1 and section 4 respectively. We also have the following abstract existence theorems of "optimal" destabilizing objects, as direct consequences of (the proof of) Theorem 2.3 combined with Kem78, Theorem 3.4] (cf. also [Rou78).

Corollary 2.6 (of Theorem [2.3). If $[X \subset \mathbb{P}]$ is Chow-unstable, it has a unique maximally destabilizing test degeneration.

Corollary 2.7. If $(X, L)$ is K-unstable, it has a unique maximally destabilizing series of test degenerations.

For the definitions of these "maximally destabilizing" objects, we will explain in section 4 that they are essentially with regard to the restricted action of $\mathrm{SL}\left(H^{0}\left(X, L^{\otimes r}\right)\right)$. Note that, if $(X, L)$ is K-unstable, $X \hookrightarrow \mathbb{P}\left(H^{0}\left(X, L^{\otimes r}\right)\right)$ is 1 Chow-unstable for sufficiently large $r \in \mathbb{Z}_{>0}$ (cf. e.g. [RT07, section 3]). Corollary 2.7 simply follows from Corollary [2.6. It would be interesting to know the behavior of Donaldson-Futaki invariants on $\Delta_{\infty}$ or to compare Corollary 2.7 with the framework of newer stability notions of [Don10] and Szze11.

It would also be interesting to discuss the relation with a similar existence statement in [Sze08, Theorem 4] which was for the toric case. He used a certain compactness property of the space of convex functions on the Delzant polytope (for which he refers to Don02, Proposition 5.2.6]).

\footnotetext{
${ }^{1}$ In the present note and other former papers of the author, the notation for the projectivization $\mathbb{P}(-)$ of vector space is the contravariant one, following Grothendieck.
} 


\section{PARAmeter SPACE}

3.1. Review of spherical building. See Tit74, AB08, for more detailed accounts of the material presented in this subsection. In this subsection, $G$ is assumed to be an arbitrary reductive algebraic group.

Definition 3.1. The spherical building $\Delta(G)$ of $G$ is an abstract simplicial complex whose vertices represent maximal (proper) parabolic subgroups and whose faces represent a finite set of maximal parabolic subgroups whose intersection is again parabolic.

Then it is known that the following holds.

Proposition 3.2 (cf. Mum65, Chapter 2, section 2]). The rational points of spherical building of $G$ has a natural correspondence with classes of one parameter subgroups as follows. We denote the center subgroup of $G$ as $Z$ and "1-ps" stands for one parameter subgroup.

$$
|\Delta(G)|_{\mathbb{Q}} \cong\left\{1-\text { ps } \lambda: \mathbb{G}_{m} \rightarrow G \text { whose image is not in } Z\right\} / \sim .
$$

Here the equivalence relation is generated by the following three relations:

(i) $\lambda^{m} \sim \lambda^{n}$ for any $m, n \in \mathbb{Z}_{>0}$.

(ii) $\lambda \sim p \lambda p^{-1}$ if $p \in(P(\lambda))_{k}$, where $P(\lambda)$ is a parabolic subgroup of $G$ whose $k$-valued points are:

$\left\{\gamma \in(G)_{k} \mid \lambda(t) \gamma \lambda\left(t^{-1}\right)\right.$ has a specialization in $G$ when $t$ specializes to 0$\}$.

(iii) $\lambda \sim \lambda \xi$ where $\xi$ is a one parameter subgroup of $Z$.

Note that by the relation (iii), and not thinking one parameter subgroup with image in $Z$, we are essentially considering a semi-simple part. Of course, fixing a maximal torus $T$, we have a natural map $|\Delta(T)|_{\mathbb{Q}} \rightarrow|\Delta(G)|_{\mathbb{Q}}$ which is indeed injective. Thus, $|\Delta(G)|_{\mathbb{Q}}$ is a union of $S(T, \mathbb{Q}):=\left(\operatorname{Hom}_{\text {alg.grp }}\left(\mathbb{G}_{m}, T\right) \otimes_{\mathbb{Z}} \mathbb{Q}\right) / \mathbb{Q}_{>0}$ which is dense in $S(T, \mathbb{R}):=\left(\operatorname{Hom}_{\text {alg.grp }}\left(\mathbb{G}_{m}, T\right) \otimes_{\mathbb{Z}} \mathbb{R}\right) / \mathbb{R}_{>0}$ (apartments) naturally homeomorphic to a sphere.

It is not compact in the usual topology (see AB08, Chapter 12] for compatible metric structure and its developments) but it is compact if we introduce a new topology as follows.

Note that a variety $G / N(T)$ parametrizes a set of maximal tori where $N(T)$ denotes the normalizer (algebraic) subgroup of a fixed maximal torus $T$ of $G$. Thus, we have a natural map $\phi: S(T, \mathbb{R}) \times(G / N(T))_{k} \rightarrow|\Delta(G)|$. Using this, we can define our new topology as its associated quotient (compact) topology of the product of the Euclidean topology on $S(T, \mathbb{R})$ and the Zariski topology on $(G / N(T))_{k}$, via $\phi$. We call it stuck topology of $|\Delta(G)|$ (or $|\Delta(G)|_{\mathbb{Q}}$, defined by restriction) in this note. We fix the above notation from now on.

3.2. Proof of Theorem 2.3. Note that we are interested in the case

$$
G=\operatorname{GL}\left(H^{0}(X, L)\right)
$$

which we assume in this subsection.

As Proposition 3.2 shows, the rational points of the spherical building $|\Delta(G)|_{\mathbb{Q}}$ represent the classes of one parameter subgroups of $\mathrm{GL}\left(H^{0}(X, L)\right)$ with respect to the equivalence relation generated by (i), (ii), (iii) which are non-trivial.

On the other hand, let us recall that each test configuration $(\mathcal{X}, \mathcal{L})$ with very ample $\mathcal{L}$ of exponent 1 and projection morphism $p: \mathcal{X} \rightarrow \mathbb{A}^{1}$ is isomorphic to the 
DeConcini-Procesi family of a one parameter subgroup ([RT07, Proposition 3.7]). More precisely, if we fix $\mathbb{G}_{m}$-equivariant trivialization $f: p_{*} \mathcal{L} \cong \mathcal{O}^{\oplus(M+1)}$ of $p_{*} \mathcal{L}$, then we can take a one parameter subgroup $\lambda_{f}$ whose corresponding DeConciniProcesi family is isomorphic to the original $(\mathcal{X}, \mathcal{L})$.

Let us fix a $\mathbb{G}_{m}$-equivariant trivialization $f$ and take the associated eigenspace decomposition of $H^{0}(X, L)$ as $H^{0}(X, L)=\oplus_{i} V_{i}$ where the eigenvalue of the action on $V_{i}$ is $t^{w_{i}}$. Then, as explained in [Don05, a few lines after Lemma 2], any other choice is given by $p(t) \circ f$ where $p \in(P(\lambda))_{k}$ and $p(t)$ is defined by $\left.p(t)\right|_{\operatorname{End}\left(V_{i}, V_{j}\right)}=$ $\left.t^{w_{j}-w_{i}} p\right|_{\operatorname{End}\left(V_{i}, V_{j}\right)}$. Note that $\lambda_{p(t) \circ f}=p \lambda_{f} p^{-1}$.

Thus, each test configuration is an equivalence class of one parameter subgroups divided by the relation (ii). And it is also easy to see that the relation (i) (resp. (iii)) corresponds to the base change, i.e. P-pre-equivalence (resp. R-equivalence) introduced in Definition 2.2. Hence, test degenerations with very ample polarization of exponent 1 correspond to one parameter subgroups of $\operatorname{GL}\left(H^{0}(X, L)\right)$ modulo (i), (ii), (iii) so that they correspond to rational points of the building $|\Delta(G)|_{\mathbb{Q}}$.

3.3. Almost trivial test configurations. In LX11, it was recently pointed out that in the definition of K-stability we should allow some non-trivial but "almost trivial" test configurations having GIT weights to vanish, or simply dismiss those test configurations of a specific type from our mind. Rigorously speaking, it is characterized by the following condition. Indeed, those should have vanishing Donaldson-Futaki invariants by the formula [Wan08, Od11.

Definition 3.3 ( $[$ Stp11, Definition 1]). A test configuration $(\mathcal{X}, \mathcal{L})$ is said to be almost trivial ${ }^{2}$ if $\mathcal{X}$ is $\mathbb{G}_{m}$-equivariantly isomorphic to the trivial test configuration, away from a closed subscheme of codimension at least 2 .

By taking the fact that they are not necessarily trivial (cf. [LX11, Example 1]) into account, K-stability is slightly reformulated as follows.

Definition 3.4 (cf. [LX11, subsection 2.2], Stp11, Definition 2]). A polarized variety $(X, L)$ is $\mathrm{K}$-stable if $\mathrm{DF}(\mathcal{X}, \mathcal{L})>0$ for all test configurations which are not almost trivial.

Now we give a partial characterization of "almost triviality" from different points of view as follows. We note that the converse does not hold in general.

Proposition 3.5. Assume $X$ is reduced, equidimensional and embedded into a projective space $\mathbb{P}\left(H^{0}\left(X, L^{\otimes r}\right)\right)$ by the complete linear system $\left|L^{\otimes r}\right|$ with some $r \in$ $\mathbb{Z}_{>0}$. Take a diagonalization of the $\mathbb{G}_{m}$-action $\lambda$ on $H^{0}\left(X, L^{\otimes r}\right)$ by the eigenvectors $X_{0}, \cdots, X_{c}, X_{c+1}, \cdots, X_{N}$ where $\mathbb{G}_{m}$ acts on $X_{i}$ by $t^{a_{i}}$. Assume that $a_{0}=\cdots=$ $a_{c}<a_{c+1} \leq \cdots \leq a_{N}$.

Under the assumptions above, if the associated test configuration is almost trivial, then $c>\operatorname{dim}(X)$ holds and the linear subspace $V\left(X_{0}, \cdots, X_{c}\right)$ of $\mathbb{P}\left(H^{0}\left(X, L^{\otimes r}\right)\right)$, where $X_{0}, \cdots, X_{c}$ vanish, does not intersect with $X$, i.e., $V\left(X_{0}, \cdots, X_{c}\right) \cap X=\emptyset$.

\footnotetext{
${ }^{2}$ It is called trivial in codimension 2 in Stp11 but we use this terminology for the following reason. That is, as Stoppa taught the author, the way of using the phrase "... in codimension 2" may be familiar to differential geometers, but it is not common for algebro-geometers and may well be confusing for them. The meanings are completely the same.
} 
Proof. First note that almost test configuration has a property that the linear projection of $X$ from $V\left(X_{0}, \cdots, X_{c}\right)$ is birational. Let us assume $V\left(X_{0}, \cdots, X_{c}\right) \cap$ $X \neq \emptyset$ and prove that the associated test configuration $\mathcal{X}_{\lambda}$ is not almost trivial by showing the non-birationality of the linear projection. Assume the contrary and take a closed point $x$ of $V\left(X_{0}, \cdots, X_{c}\right) \cap X$, and denote the linear projection from $x$ by $\pi$. As the linear projection from $V\left(X_{0}, \cdots, X_{c}\right)$ is a composite of the linear projection from $x$ and another linear projection, it is enough to show nonbirationality of $\left.\pi\right|_{X}$.

Note that $\pi$ naturally extends to a morphism from the blow up of $X$, i.e. $\tilde{\pi}: B l_{x}(X) \rightarrow \pi_{*}(X)$, where $\pi_{*}(X)$ denotes the reduced scheme whose underlying space is the closure of the image of the linear projection $\pi$ of $X$. Since $x \in X, B l_{x}(X) \in B l_{x}\left(\mathbb{P}\left(H^{0}\left(X, L^{\otimes r}\right)\right)\right)$ intersects with the exceptional divisor $E$ of $\varphi: B l_{x}\left(\mathbb{P}\left(H^{0}\left(X, L^{\otimes r}\right)\right)\right) \rightarrow \mathbb{P}\left(H^{0}\left(X, L^{\otimes r}\right)\right)$. Note that if $\left.\pi\right|_{X}$ was birational, it would imply the following equality of intersection numbers:

$$
\left(\mathcal{O}_{\mathbb{P}\left(H^{0}\left(X, L^{\otimes r}\right)\right)}(1)^{\cdot \operatorname{dim}(X)} \cdot X\right)=\left(\mathcal{O}(1)^{\cdot \operatorname{dim}(X)} \cdot \pi_{*}(X)\right),
$$

where $\mathcal{O}(1)$ on the right hand side means the tautological ample line bundle on the projective space to which the linear projection $\pi$ maps. It is because both equal $\left(\mathcal{O}(1)^{\prime \cdot \operatorname{dim}(X)} \cdot \pi_{*}^{\prime}(X)\right)$ where $\pi^{\prime}$ is a linear projection from $x^{\prime} \in \mathbb{P}\left(H^{0}\left(X, L^{\otimes r}\right)\right)$ which is sufficiently close to $x$ so that $\pi^{\prime}$ is also birational, and $\mathcal{O}(1)^{\prime}$ is the tautological ample line bundle on the projective space to which the linear projection $\pi^{\prime}$ maps.

The right hand side of 1 equals $\left.\left.\left(\left(\varphi^{*}\left(\mathcal{O}_{\mathbb{P}\left(H^{0}(X, L \otimes r\right.}\right)\right)(1)\right)(-E)\right)^{\cdot \operatorname{dim}(X)} \cdot B l_{x}(X)\right)$ by the projection formula with respect to $\pi$ and our assumption of birationality of $\left.\tilde{\pi}\right|_{X}$. On the other hand, it should be smaller than the left hand side by a simple volume comparison, since $E$ should intersect with $B l_{x}(X)$ in this case. This gives a contradiction. Thus, the resulting test configuration should not have a central number which is birational to original $X$.

Secondly we will prove that $c \leq \operatorname{dim}(X)$ implies that the corresponding test configuration cannot be an almost trivial test configuration as well. Note that if the linear projection from $V\left(X_{0}, \cdots, X_{c}\right)$ is birational, $c=\operatorname{dim}(X)$ follows from it by comparing dimensions. If the linear projection was birational to a projective space, this means $X \in \mathbb{P}\left(H^{0}\left(X, L^{\otimes r}\right)\right)$ is a degree 1 closed subscheme by a projection formula. However that degree 1 condition implies it is a linear subspace which contradicts with the non-degeneracy condition of the embedding. Hence the projection from $V\left(X_{0}, \cdots, X_{c}\right)$ is not birational. This completes the proof.

In other words, if a test configuration which corresponds to the one parameter subgroup $\lambda$ above is almost trivial, then its associated tag ideal $J \subset \mathcal{O}_{X \times \mathbb{A}^{1}}$ (cf. Od11] is $\left(t^{a_{c}}\right)$. Indeed, recall that $\mathcal{J}$ was defined as

$$
\mathcal{J O}_{\mathbb{P} \times \mathbb{A}^{1}(1)}=\sum_{1 \leq j \leq N, a_{i} \leq j} \mathcal{O}_{\mathbb{P}} X_{i} t^{j}
$$

Conversely, if $\mathcal{J}$ is of the form $\left(t^{M}\right)$ with some $M \in \mathbb{Z}_{>0}$, the natural dominating morphism from $B:=B l_{\mathcal{J}}\left(X \times \mathbb{A}^{1}\right)\left(\cong\left(X \times \mathbb{A}^{1}\right)\right)$ to $\mathcal{X}$ is an isomorphism away from a closed subscheme of $\mathcal{X}$ with codimension at least 2 . Recall that this is a characterization of the almost triviality.

Therefore, given a test configuration which is not almost trivial, the associated tag ideal $\mathrm{J}$ is not of the form $\left(t^{M}\right)$ for some $M \in \mathbb{Z}_{>0}$ or, if it is of such a form, $\mathcal{B} \rightarrow \mathcal{X}$ is not isomorphic in codimension 1. For the latter case, we have $\operatorname{DF}(\mathcal{X}, \mathcal{M})>0$ 
by the arguments of [RT07, section 5] or the formula for the Donaldson-Futaki invariant Od11.

On the other hand, once a semi-test configuration of blow-up type $\left(\mathcal{B}:=B l_{\mathcal{J}}(X \times\right.$ $\left.\left.\mathbb{A}^{1}\right), \mathcal{L}^{\otimes r}(-E)\right)$ is given, we can construct a contracted (ample) test configuration (Proj $\left.\oplus_{k \geq 0} H^{0}\left(X \times \mathbb{A}^{1}, \mathcal{J}^{k} p_{1}^{*} L^{\otimes r k}\right), \mathcal{O}(1)\right)$ with obviously the same DonaldsonFutaki invariant.

In sum, we have the following, which is a corrected proposition of Od11].

Corollary 3.6 (cf. Od11, Corollary 3.11]). Assume $X$ is Gorenstein in codimension 1. Then a polarized variety $(X, L)$ is K-stable (in the sense of Definition 3.4) if and only if for all semi-test configurations of the blow-up type in $\operatorname{Od11}$ (i.e., $(\mathcal{B}=$ $\left.B l_{\mathcal{J}}\left(X \times \mathbb{A}^{1}\right), \mathcal{L}^{\otimes r}(-E)\right)$ with $r \in \mathbb{Z}_{>0}$, flag ideal $\mathcal{J}$ which is not of the form $\left(t^{N}\right)$ and $\mathcal{B}$ Gorenstein in codimension 1$)$, the Donaldson-Futaki invariant is positive.

Originally, we claimed the corresponding statement for original K-stability Don02 which does not work since he ignored the case $\mathcal{J}=\left(t^{N}\right)$ so that the blow-up morphism $\mathcal{B} \rightarrow X \times \mathbb{A}^{1}$ is trivial. The proof works for proving the above statement. We apologize for this inaccuracy.

Thus, with this slightly modified K-stability notion and Corollary 3.6, the results of Od11] and the sequels are justified in the naturally corrected form, or partially work without change.

We explain a locus of the spherical building, inside which these pathologies occur.

Definition 3.7. Assume $T$ is a maximal torus of $G=\mathrm{GL}\left(H^{0}\left(X, L^{\otimes r}\right)\right)$ and take the corresponding diagonalizing basis $X_{0}, \cdots, X_{N}$ of $H^{0}\left(X, L^{\otimes r}\right)$. In the rational sphere $S(T, \mathbb{Q}):=\left(\right.$ Hom $\left._{\text {alg.grp }}\left(\mathbb{G}_{m}, T\right) \otimes \mathbb{Q}\right) / \mathbb{Q}_{>0}$, we define a subset

$$
S^{\prime}(T, \mathbb{Q}):=\left\{\left(a_{i}\right) \mid V\left(\left\{X_{j} \mid a_{j}=\min _{i}\left\{a_{i}\right\}\right\}\right) \cap X=\emptyset\right\} .
$$

Note that $S^{\prime}(T, \mathbb{Q})$ has a dense complement as for each $\left(a_{i}\right) \in S^{\prime}(T, \mathbb{Q})$ we have $\#\left\{j \mid a_{j}=\min _{i}\left\{a_{i}\right\}\right\}>1$. It is because the corresponding linear subspace should not be of codimension 1 , as $X \subset \mathbb{P}\left(H^{0}\left(X, L^{\otimes r}\right)\right)$ is non-degenerate.

Corollary 3.8 (of Proposition 3.5). In the geometric realization of the spherical building $\Delta(G)$, almost trivial test degenerations are parametrized by $\phi\left(\cup_{T} S^{\prime}(T, \mathbb{Q})\right)$ whose complement $\left(\right.$ in $\left.|\Delta(G)|_{\mathbb{Q}}\right)$ is dense.

\section{Weight FUnCTION}

We give the definitions of normalized Chow weights and optimal destabilizing test degeneration as follows. See Mum65, Chapter 1, 2] to review basics of GIT weights. Assume $G:=\operatorname{SL}\left(H^{0}\left(X, L^{\otimes r}\right)\right)$ in this section and fix a maximal torus $T$ of $G$. With a Weyl group invariant norm $|\cdot|$ on $\operatorname{Hom}_{\text {alg.grp }}\left(\mathbb{G}_{m}, T\right) \otimes_{\mathbb{Z}} \mathbb{R}$, denote the normalized GIT weight function by $\nu_{T}:=\frac{\mu_{T}}{|\cdot|}$, where $\mu_{T}$ is the usual GIT weight function restricted to the set of one parameter subgroups of $T$. Recall that $\nu_{T}:|\Delta(T)|_{\mathbb{Q}} \rightarrow \mathbb{R}$ can be naturally extended to $\nu$ on whole $|\Delta(G)|_{\mathbb{Q}}$ by taking conjugate which sit in $T$ for each one parameter subgroup. Note that $\Delta(G)=\Delta\left(\operatorname{SL}\left(H^{0}\left(X, L^{\otimes r}\right)\right)\right)$ can be and will be naturally identified with $\Delta\left(\mathrm{GL}\left(H^{0}\left(X, L^{\otimes r}\right)\right)\right)$ by their definitions.

Definition 4.1. For a given embedded projective variety $[X \subset \mathbb{P}]$ and one parameter subgroup $\lambda: \mathbb{G}_{m} \rightarrow \operatorname{SL}\left(H^{0}(\mathbb{P}, \mathcal{O}(1))\right)$, its normalized Chow weight means $\nu(\bar{\lambda} ; \operatorname{Chow}(X \subset \mathbb{P}))$, where $\operatorname{Chow}(X \subset \mathbb{P})$ means the associated Chow point.

One of the main issues of this note is the existence of the following object. 
Definition 4.2. Assume $[X \subset \mathbb{P}]$ is Chow-unstable. Then, its maximally destabilizing test degeneration means the test degeneration which corresponds to a point of $|\Delta(G)|_{\mathbb{Q}}$ representing one parameter subgroup in $\operatorname{SL}\left(H^{0}\left(X, L^{\otimes r}\right)\right)$ which has the minimum (so negative) normalized Chow weight.

Note that Corollary 2.6, which states its existence and uniqueness, simply follows from Rou78, Kem78 combined with Theorem 2.3 of this note.

Now we briefly explain the continuity property of normalized Chow weights (Theorem 2.5).

Proof of Theorem 2.5. Recall the following lemma due to Mumford.

Lemma 4.3 (cf. [Mum65, Chapter 2, Proposition 2.14]). With finite linear functions on $\mathrm{Hom}_{\text {alg.grp }}\left(\mathbb{G}_{m}, T\right) \otimes_{\mathbb{Z}} \mathbb{R}$ with rational coefficients $l_{1}, \cdots, l_{m}$, and a map $I: G \rightarrow\{$ non-empty subsets of $\{1, \cdots, m\}\}$, the weight function $\mu$ is of the form

$$
\mu_{g T g^{-1}}(\lambda)=\max \left\{l_{i}(\bar{\lambda}) \mid i \in I(g)\right\} .
$$

Here, I satisfies "lower semicontinuity" in the sense that for any $J \subset\{1, \cdots, m\}$, $\{g \in G \mid J \subset I(g)\}$ is open.

Although the last sentence is not explicitly written in Mumford's exposition, it is essentially proved there. See also Kem78. (He used his own terminology "states" which corresponds to our map I.) The continuity of the right hand side implies continuity of normalized Chow weights $\nu$ on $|\Delta(G)|_{\mathbb{Q}}$ and, moreover, the last sentence implies lower semi-continuity with respect to the stuck topology.

\section{ACKNowledgements}

The author is grateful to Professor Shigefumi Mori for his helpful advice. The author would also like to thank Chi Li, Yoshiki Oshima, Jacopo Stoppa, Song Sun and Gabor Szekelyhidi for their helpful comments.

\section{REFERENCES}

[AB08] Peter Abramenko and Kenneth S. Brown, Buildings, Theory and applications, Graduate Texts in Mathematics, vol. 248, Springer, New York, 2008. MR2439729 (2009g:20055)

[Don02] S. Donaldson, Scalar curvature and stability of toric varieties, J. Diff. Geom. 62 (2002), no. 2, 289-349. MR.1988506(2005c:32028)

[Don05] S. K. Donaldson, Lower bounds on the Calabi functional, J. Differential Geom. 70 (2005), no. 3, 453-472. MR2192937 (2006k:32045)

[Don10] S. Donaldson, Stability, birational transformations and the Kähler-Einstein problem, Surv. Differ. Geom., 17, Int. Press, Boston, MA, 2012. MR3076062

[GSZ11] T. L. Gomez, I. Sols, and A. Zamora, A GIT interpretration of the Harder-Narasimhan filtration, arXiv:1112.1886.

[Kem78] George R. Kempf, Instability in invariant theory, Ann. of Math. (2) 108 (1978), no. 2, 299-316, DOI 10.2307/1971168. MR506989 (80c:20057)

[LX11] C. Li and C. Xu, Special test configurations and K-stability of $\mathbb{Q}$-Fano varieties, arXiv:1111.5398.

[Mum65] David Mumford, Geometric invariant theory, Ergebnisse der Mathematik und ihrer Grenzgebiete, Neue Folge, Band 34, Springer-Verlag, Berlin, 1965. MR0214602 (35 \#5451)

[Od11] Y. Odaka, A generalization of the Ross-Thomas slope theory, Osaka J. Math. 50 (2013), no. 1, 171-185. MR.3080636

[Rou78] Guy Rousseau, Immeubles sphériques et théorie des invariants (French, with English summary), C. R. Acad. Sci. Paris Sér. A-B 286 (1978), no. 5, A247-A250. MR0506257 $(58 \# 22063)$ 
[RT07] Julius Ross and Richard Thomas, A study of the Hilbert-Mumford criterion for the stability of projective varieties, J. Algebraic Geom. 16 (2007), no. 2, 201-255, DOI 10.1090/S1056-3911-06-00461-9. MR2274514 (2007k:14091)

[Stp11] J. Stoppa, A note on the definition of K-stability, arXiv:1111.5826.

[Sze08] Gábor Székelyhidi, Optimal test-configurations for toric varieties, J. Differential Geom. 80 (2008), no. 3, 501-523. MR2472481(2009m:53179)

[Sze11] G. Szekelyhidi, Filtrations and Test-configurations, arXiv:1111.4986.

[Tia97] Gang Tian, Kähler-Einstein metrics with positive scalar curvature, Invent. Math. 130 (1997), no. 1, 1-37, DOI 10.1007/s002220050176. MR1471884(99e:53065)

[Tit74] Jacques Tits, Buildings of spherical type and finite BN-pairs, Lecture Notes in Mathematics, Vol. 386, Springer-Verlag, Berlin, 1974. MR0470099 (57 \#9866)

[Wan08] X. Wang, Height and GIT weight, Math. Res. Lett. 19 (2012), no. 4, 909-926. MR3008424

Department of Mathematics, Kyoto University, Kyoto, 606-850, JAPAN

E-mail address: yodaka@math.kyoto-u.ac.jp 